\title{
Evaluation of the laser-flash method and its errors for determining of materials thermal diffusivity at high temperatures
}

\author{
Mark D. Katz ${ }^{1, *}$, Ilija M. Katz ${ }^{1}$ \\ ${ }^{1}$ National Research Tomsk Polytechnic University, 634050 Tomsk, Russia
}

\begin{abstract}
The article deals with evaluation of determining constructional materials thermal characteristics systematic errors by laser-pulse method under high temperature conditions. The article gives a detailed analysis of the influence of heat exchange with surrounding air on systematic errors. It is shown that laser pulse method can be used for calculation of thermal diffusivity and thermal capacity of the metals and alloys with high thermal diffusivity for temperatures not exceeding $1173 \mathrm{~K}$.
\end{abstract}

\section{Introduction}

Applying advanced materials for engineering systems requires knowledge of their thermal characteristics [1]. For this purpose, nowadays, different methods of thermal characteristics (TC) determination are used. But for TC determination at high temperature these methods become more complicated. More expensive experimental facilities and measurement systems should be used in this case. Also, a preparation time and duration of experiments are increasing too $[2,3]$. In light of this, more simple methods of thermal characteristics determination should be used. One of these methods is a laser flash method (LFA) [4-8]. According to LFA, the maximum temperature $\left(T_{\max }\right)$ of a sample heating and heating time $\left(\tau_{05}\right)$ of the sample to half of $T_{\max }$ are defined using experimental time-based temperature data of the reverse ("cold") surface of the sample.

The thermal diffusivity $a$ of the material can be found through approximate solution of the one-dimensional heat conduction problem for an infinite plate [4]:

$$
a=0.1388 L^{2} / \tau_{0.5}
$$

where $L$ is trickiness of the sample.

For equation (1) solution values $T_{\max }$ and $\tau_{05}$ must be known.

Conditions for TC reliable determination at low temperature band and estimation errors of it are described in a fairly large number of papers [4-10]. TC at medium and high temperatures (no greater than $1173 \mathrm{~K}$ ) can be determined only in experimental way. But

\footnotetext{
*Corresponding authors: riki@tpu.ru
} 
these experiments are carried out under conditions of active heating of a sample. Under such conditions TC of the sample is very hard to detect $[1,5,6]$. So, at the first stage of the TC determination process it might be useful to carry out theoretical experiment. Results of this experiment could be used for planning of physical experiment. In this case, the determination of TC by theoretical way is based on mathematical modelling of thermal conductivity process in plane sample, under relevant conditions for LFA [9,10].

Comparison of calculated TC by mathematical modelling with TC assumed during this simulation in $[9,10]$ is allowed to evaluate influence various factors to errors of TC determination. The approach $[9,10]$ is used for determination of TC of highly heatconductive materials at medium and high temperatures (no greater than $1173 \mathrm{~K}$ ).

The aim of the present work is theoretical analysis of the errors in determining the TC of materials for engineering systems at medium and high temperatures by the LFA.

\section{Statement of the problem}

During the LFA development it was supposed, that there is not abstraction of heat form the sample surface to the outdoor environment [4, 5]. As was shown in [9, 10], such assumption doesn't influence the TC of the sample at low temperature. In the case under consideration, when temperature is over $1000 \mathrm{~K}$, heat-gravitational convection will significantly affect the heat flow pattern of a sample during the experiment. This phenomenon also has an impact on the further results of measurement of $T_{\max }$ and $\tau_{05}$. Therefore, it is necessary to take into account abstraction of heat from the sample surface, which caused by natural convection. Different approaches to the solution of this task are used.

In particular, this process can be described by a conjugate convective heat transfer problem [11, 12]. However, implementation of algorithms [11, 12] requires the significant time resources. To simplify them, integral parameters (surface heat-transfer coefficients) should be used.

Simulation of heating processes during laser pulse exposure with thermal flux density $q$ and pulse time $\Delta t$ were made for case of horizontal placed thin plate. Abstraction of heat from the sample surface, which caused of the natural convection, was taken into account during this simulation.

Simulation was made for typical construction materials: stainless steel $12 \mathrm{H} 18 \mathrm{~N} 10 \mathrm{~T}$, titanium and silicone carbide at temperature band 673-1173 K.

Heat transfer was modeled under the following assumptions: 1) the materials are gray and their self-radiation is small in comparison with the external radiant flux; 2) thermophysical parameters of materials at the temperature band of heating by laser pulse correspond to the temperature of sample preheating; 3 ) cooling-down of the sample is owing to abstraction of heat from the front and back surface of the sample, which caused the natural convection; 4) linear dilatation of the sample during temperature rise was not taken into account.

The first assumption doesn't restrict the problem statements and real conditions of heat interchange through the material.

The mathematical model that corresponds to the adopted formulation of the problem involves one-dimensional nonstationary differential energy equations for thin plate with constant thermal flow $q$ directed to the heated surface during laser pulse time $\Delta t$ and convective heat transfer to the outdoor environment (fig. 1). 


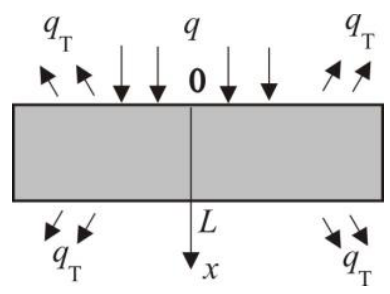

Fig. 1. Scheme of the area of solution.

The mathematical model includes nonstationary partial differential heat-transfer equation (2) with boundary conditions (3)-(5) and initial condition (6):

$$
\begin{array}{lc} 
& c \rho \frac{\partial T}{\partial t}=\lambda\left(\frac{\partial^{2} T}{\partial x^{2}}\right), \quad 0<x<L \\
x=0: & -\lambda \frac{\partial T}{\partial x}=q+\alpha\left(\mathrm{T}_{e}-\mathrm{T}\right), \quad \alpha>0, \quad \Delta t>t>0 ; \\
x=0: & -\lambda \frac{\partial T}{\partial x}=\alpha\left(\mathrm{T}_{e}-\mathrm{T}\right), \quad \alpha>0, \quad t>\Delta t ; \\
x=L: & \lambda \frac{\partial T}{\partial x}=\alpha\left(\mathrm{T}_{e}-\mathrm{T}\right), \quad t>0 ; \\
t=0: & T=T_{0},
\end{array}
$$

where $c$ - heat capacity, $\mathrm{J} /(\mathrm{kg} \cdot \mathrm{K}) ; \rho$ - the density, $\mathrm{kg} / \mathrm{m}^{3} ; T$ - temperature, $\mathrm{K} ; t$ - time, $\mathrm{s} ; \Delta t$ - the duration of the laser pulse, s.; $q$ - radiation flux density directed to the heated sample surface, $\mathrm{W} / \mathrm{m}^{2} ; T_{0}$ - initial value of the current temperature, $\mathrm{K} ; T_{e}$ - outdoor environment temperature, $\mathrm{K} ; \alpha$ - heat-exchange to outdoor environment coefficient, $\mathrm{W} /\left(\mathrm{m}^{2} \mathrm{~K}\right)$.

The above mentioned nonlinear boundary value problem was solved by the method of finite differences using an iteration algorithm, developed for the numerical solution of hightemperature heat-transfer problems under conditions of active physicochemical transitions of high-energy condensate liquid substances [13]. The reliability of the results of the numerical modeling was checked by comparison them with experimental data [14]. Also conservatism of used difference scheme according to procedure discussed in [13] was analyzed.

\section{Results and discussion}

Simulation of heat transfer process was made for stainless steel 12H18N10T, titanium and silicone carbide at temperature band $673-1173 \mathrm{~K}$ under the following conditions: $q=3.5 \cdot 10^{7} \ldots 6.6 \cdot 10^{7} \mathrm{~W} / \mathrm{m}^{2}, \Delta t=1.0 \mathrm{~ms}, T_{0}=300 \mathrm{~K}$. Numerical values of TC were adopted in accordance with the papers $[15,16]$. Samples thickness was chosen $5 \mathrm{~mm}$ according to conditions in [9] to minimize error of TC determination method. 
During the simulation a time step was chosen $1 \mathrm{~ms}$. The calculations were performed with moves in space to 2.5 microns. Heat-exchange to outdoor environment coefficient $\alpha$ varied within the limits from 0 to $5 \mathrm{~W} / \mathrm{m}^{2} \mathrm{~K}$.

The values of the $T_{\max }$ and $\tau_{05}$ were found based on the results of the numerical solution of the problem (2-7). The thermal diffusivity was calculated by (1).

Errors of TC determination by LFA method under heat-exchange to outdoor environment and convective heat transfer in the sample conditions were defined by comparing reference value of thermal diffusivity $[16,17]$ with calculated one.

The calculated thermal diffusivity for steel $12 \mathrm{H} 18 \mathrm{~N} 10 \mathrm{~T}$ was also weighed against experimental data [14] at high temperatures $T_{0}$. Results of this comparison showed, that the difference between these values didn't exceed $5 \%$. That's why we can conclude that mathematical model (2)-(7) is valid for simulation of heat transfer in a plate, heated by short laser pulse at high-temperature region (no greater than $1173 \mathrm{~K}$ ). Also this mathematical model can be used for TC determination of construction materials at temperature band $673-1173 \mathrm{~K}$.

During calculation of sample temperature pattern, heated to high temperature, phenomenon of heat abstraction from the sample surface at plate borders should be taken into account, because heat losses are increasing with rising of heated and "cold" surfaces temperature. That, in turn, led to additional errors of $T_{\max }$ and $\tau_{05}$ determination.

Figure 2 presents the dependences of the errors in the determination of the thermal diffusivity $\delta_{a}$ on the heat-exchange to outdoor environment coefficient $\alpha$ under conditions of natural convection. It was established that $\delta_{a}$ depends not only on level of heat abstraction, but also it depends on material thermal conductivity.

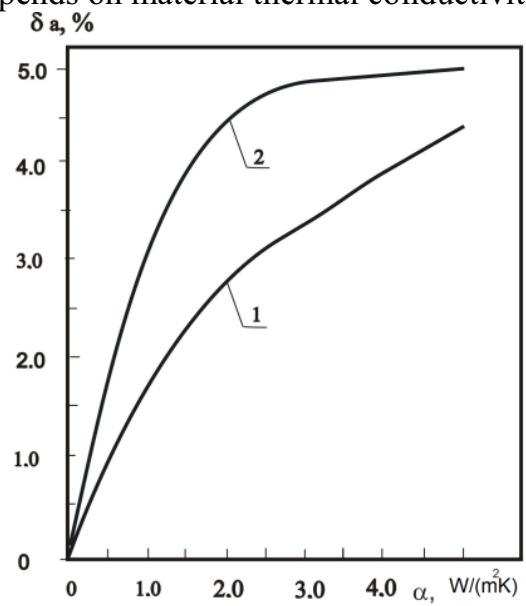

Fig. 2. Errors in determination of the thermal diffusivity $\delta_{a}$ the convective heat transfer coefficient at heat temperature $873 \mathrm{~K}: 1$ - titanium, 2 - silicone carbide.

This fact is shown in fig.2, the error in the determination of the thermal diffusivity $\delta_{a}$ of silicone carbide exceeds errors of other materials. It mainly owing to relatively low thermal conductivity of silicone carbide as against titan thermal conductivity. As shown in fig. 2, under conditions of short heating pulse, steel sample doesn't warm fast. Temperature of it surface doesn't exceed $899.5 \mathrm{~K}$. The heat abstraction process form the sample surface to the outdoor environment (air) occurs at heat difference about $600 \mathrm{~K}$. During the heating of silicone carbide the surface temperature is higher $(939 \mathrm{~K})$ and the heat difference is 
about $639 \mathrm{~K}$. Stored during the pulse duration heat at thin surficial region of front sample surface doesn't transfer to the "cold" surface, which peak temperature is used as governing characteristic for $a$ calculation. This heat is transferred to the outdoor environment.

The results of the simulation give good reason to draw conclusions that the laser pulse method [4] can be used for determination of TC of materials (metals and alloys) for engineering systems at medium and high temperatures (no greater than $1173 \mathrm{~K}$ ). For experimental determination TC of materials with low thermal conductivity measurements should be done in special closed boxes. The temperature in these boxes must be close to the sample heating temperature. It's necessary to provide the conditions of low heat losses of the sample.

\section{Conclusion}

An analysis of the systematic errors in the determination of the thermophysical characteristics of materials (metals and alloys) for engineering systems at medium and high temperatures (no greater than $1173 \mathrm{~K}$ ) has been made by of the mathematical simulation of heat transfer processes.

Applying of LFA for TC determination under condition of convective transfer to environment and its errors were evaluated.

It was established, that LFA method can be used for TC determination of highly heatconductive materials under high temperature conditions.

\section{References}

1. E.E. Son, High Temp. 51, 3 (2013)

2. M.V. Ilichev, V.B. Mordinskii, D.V. Tereshenok, High Temp. 53, 2 (2015)

3. Z.A. Musaeva, V.E. Peleckii, High Temp. 40, 6 (2002)

4. W.J. Parker, R.J. Jenkins, C.P. Butler, J. Appl. Phys. 32, 9 (1961)

5. M. Akoshima, T. Baba, Int. J. Thermophys. 27, 4 (2006)

6. O. Altun, B. Erhan, A. Kalemtas, J. Achievements Mater. Manuf. Eng. 30, 2 (2008)

7. D. Moskal, J. Martan, V. Lang, Int. J. Heat Mass Transf. 102, 1 (2016)

8. S. Lee, D.H. Kim, High Temp. High Pressures, 45, 5 (2016)

9. G.V. Kuznetsov, M.D. Kats, Meas. Techn. 55, 4 (2012)

10. G.V. Kuznetsov, M.D. Kats, Meas. Techn. 53, 6 (2010)

11. G.V. Kuznetsov, V.A. Sheremet, Computational Thermal Sciences, 1, 3 (2009)

12. G.V. Kuznetsov, V.A. Sheremet, Fluid Dynamics, 41, 6 (2006)

13. O.V. Vysokomornaya, G.V. Kuznetsov, P.A. Strizhak, Chem. Phys. 30, 8 (2011)

14. S.V. Stankus, I.V. Savchenko, A.V. Baginskii, High Temp. 46, 5 (2008)

15. V.S. Chirkin, Thermal-physical properties of materials for nuclear engineering: Manual (Atomizdat, Moscow, 1968) [in Russian]

16. A.S. Zubchenko, Steels and alloys grade guide (Machinostroenie, Moscow, 2003) [in Russian] 